\title{
Mariposas en un fragmento de bosque seco tropical en Montes de María (Colombia).
}

\section{Butterflies in tropical dry forest fragment at Montes of Maria (Colombia).}

\author{
Yarlenis L. Mercado-Gómez a* \\ Jorge D. Mercado-Gómez ${ }^{b}$ \\ Carlos E. Giraldo - Sánchez c
}

Fecha de Recepción: 12 - dic. - 2017.

Fecha de Aceptación: 30 - abr. - 2018.

\section{Resumen}

La riqueza de las mariposas diurnas en los Montes de María (Serranía de Coraza), fue caracterizada a través de tres transectos lineales de $600 \mathrm{~m}$. Ocho parcelas circulares de $15 \mathrm{~m}$ de radio se emplearon al interior de cada parcela, recolectando los especímenes con red entomológica y trampas Van Someren Rydon. Se realizaron curvas de rango-abundancia para comparar la composición, abundancia y uniformidad de las especies en cada transecto; además el número esperado de especies fue calculado a través de una curva de rarefacción y extrapolación-interpolación. En total se recolectaron 301 individuos agrupados en 4 familias, 13 subfamilias, 22 tribus, 44 géneros y 55 especies. Nymphalidae presentó la mayor abundancia (235) y riqueza (37); mientras que Biblidinae (120 individuos) y Hamadryas februa (56 individuos) son la subfamilia y especie más abundantes. Los resultados sugieren que los bosques secos de Coraza, a pesar de su degradación por las actividades antrópicas, poseen gran riqueza de mariposas. Este estudio demuestra la importancia de seguir realizando estudios de composición de mariposas que permitan conocer no solo la estructura de estas comunidades, sino también para incrementar el conocimiento de la entomofauna de los bosques secos dentro de la reserva forestal Serranía de Coraza.

Palabras Claves: Montes de María, Colosó, riqueza, composición, lepidoptera.

a Maestría en Biología. Grupo Evolución y Sistemática Tropical. Universidad de Sucre, Carrera 28 No. 5-27. Sincelejo, Colombia. Yarlenis29@gmail.com.

b Grupo Evolución y Sistemática Tropical. Departamento de Biología y Química. Universidad de Sucre, Carrera 28 No. 5-27. Sincelejo, Colombia. jorge.mercado@unisucre.edu.co.

c Grupo de Investigación de Sanidad Vegetal. Universidad Católica del Oriente, Rionegro-Colombia.

* Autor para correspondencia. 


\begin{abstract}
The richness of diurnal butterflies in the Montes de Maria (Serranía de Coraza) was characterized using linear transects of $600 \mathrm{~m}$. long. Inside each transects eight circular plots of $15 \mathrm{~m}$ of radius were established. To collected butterflies, we used entomological network and Van Someren Rydon traps. To compare the composition, abundance and uniformity of the species in each transect and to establish if the samples were significant, range-abundance curves were performed. In addition, to calculated the expected number of species curve of rarefaction and extrapolation-interpolation were made. In total, 301 individuals were collected belongs to 4 families, 13 subfamilies, 22 tribes, 44 genera and 55 species. Nymphalidae have the highest abundance (235) and richness (37), while Biblidinae (120 individuals) and Hamadryas februa (56 individuals) were more abundant subfamily and species. Results suggest that tropical dry forests of Coraza, despite the anthropic impact, have a great diversity butterflies' species. Herein we show the importance of following studying the butterfly composition and diversity that allows, not only know the structure of these communities, also to increase the knowledge inside tropical dry forest entomofauna of Serrania Coraza reserve.
\end{abstract}

Keywords: Montes de María, Colosó, richness, composition.

\section{INTRODUCCIÓN}

El bosque seco tropical (bs-t), ha sido definido como uno de los ecosistemas de la franja tropical en los cuales la precipitación anual no supera los $1,800 \mathrm{~mm}$ y se presenta una marcada estacionalidad durante dos a seis meses en los cuales la precipitación no supera los $100 \mathrm{~mm}$ por mes [1]. Estas condiciones han permitido que estos bosques sean considerados como uno de los ecosistemas más complejos e interesantes en la región neotropical, producto de las adaptaciones que han sufrido sus especies a tan particulares y extremas variaciones del clima [2].

En Colombia los bosques secos están distribuidos principalmente sobre los valles interandinos y en la región Caribe [3] con una extensión $8,882,854$ ha [4], dentro de las cuales recientemente se estableció que el $34.23 \%$ están siendo utilizadas para pasturas, $28.25 \%$ para cultivos, $15.0 \%$ en otros usos y solo el $22.50 \%$ son considerados como bosques con cobertura natural [3]. En otras palabras, estos bosques actualmente se encuentran en alto grado de amenaza [3]. La región Caribe, aparentemente presenta los fragmentos de bosque seco de mayor tamaño y en mejor estado de conservación. No obstante, García, et al. [3] menciona que solo el 37,97\% de las 533.099 ha, que históricamente estaban conformadas por fragmentos de bosque seco, aún permanecen como áreas naturales. Incluido dentro de estos fragmentos se encuentran los Montes de María (localizados entre los departamento de Sucre y Bolívar), catalogado por Pizano, et al.
[5] como uno de las zonas en mejor estado de conservación. Empero, análisis recientes muestran que estos bosques han sido fragmentados y degradados en los últimos años $[6,7]$. Asimismo, otro de los problemas que actualmente afectan estos bosques es la falta de conocimiento sobre la diversidad de varios grupos taxonómicos como los insectos. Las mariposas, por ejemplo son un grupo poco explorado en estos Montes [8].

Las mariposas son un grupo de insectos de suma relevancia, ya que han sido sugeridas como indicadores útiles de los cambios a nivel del paisaje, debido a su visibilidad, fácil identificación, rápida reproducción, su estrecha relación con factores físicos y su sensibilidad a los cambios ambientales [9, 10]. Además, cumplen con la mayoría de los criterios que debe tener un taxón para ser considerado un indicador del estado de preservación o alteración de los bosques y la biodiversidad de los ecosistemas [10]. Dentro de estos se incluyen: la diversidad y distribución están bien descritas; son relativamente fáciles de muestrear, existen guías de campo accesibles para su identificación, su taxonomía es relativamente estable, son abundantes y diversas en muchos ecosistemas [11].

Con el fin de contribuir al conocimiento global de la biodiversidad y por consecuente en la conservación de los bosques secos en esta región del país, el objetivo de la presente investigación, fue caracterizar la riqueza de mariposas diurnas en la Serranía de Coraza (Montes de María), focalizando principalmente en cinco familias: 
Nymphalidae, Pieridae, Papilionidae, Riodinidae y Lycaenidae.

\section{MATERIALES Y MÉTODOS}

\section{Área de estudio}

Los Montes de María se encuentran ubicados en la parte nororiental del departamento de Sucre, sobre la llanura del Caribe colombiano [12]. En años recientes, Montes de María se ha visto afectado por la expansión de la frontera agrícola y ganadera, motivo por el cual se establecieron dos reservas naturales: Reserva Forestal Protectora Serranía de Coraza y Montes de María (dpto. de Sucre) y el Santuario de Fauna y Flora Los Colorados (dpto. de Bolívar) [12]. La Reserva Forestal Protectora Serranía de Coraza se ubica en la jurisdicción del municipio de Colosó [13] (Figura 1). Esta reserva presenta temperaturas entre los $25-28^{\circ} \mathrm{C}$, precipitación de $896-1233$ $\mathrm{mm}$ anual, con un periodo estacional seco (Noviembre-Febrero) y $83.5 \%$ de humedad relativa promedio [12, 14].

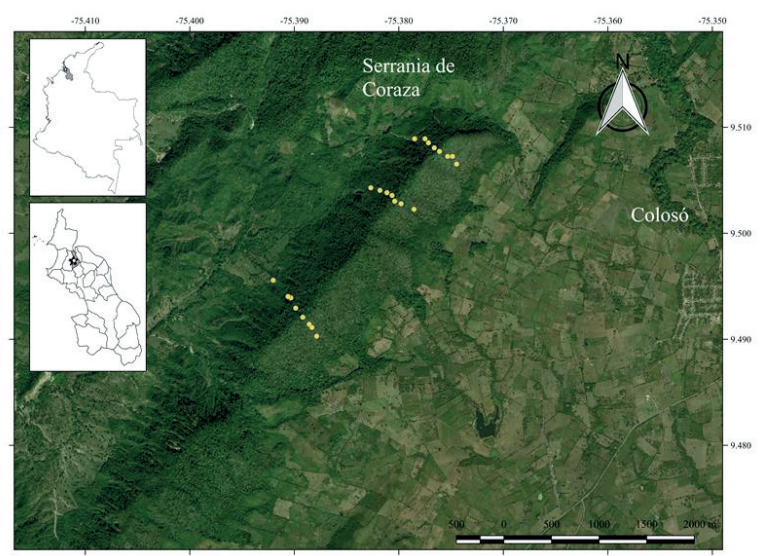

Figura 1. Localización geográfica de la Reserva Forestal Protectora Serranía de Coraza y las tres réplicas de muestreo al interior del bosque.

Respecto a la zona de vida, corresponde a bosque seco tropical (bs-t), según el sistema de clasificación de Holdrigde [15], cuya vegetación presenta grupos taxonómicos característicos de las familias Fabaceae, Malvaceae, Meliaceae, Sapindaceae, Capparaceae, Rubiaceae y Cactaceae. No obstante, como gran parte del departamento de Sucre, estos fragmentos de bosques se encuentran en un mosaico de vegetación conformado por bosques, cultivos y zonas de pastoreo-ganadería [7].

\section{Fase de campo}

Para establecer la riqueza de mariposas diurnas, fueron diseñados tres transectos lineales de aproximadamente $600 \mathrm{~m}$ de largo, en los cuales se establecieron ocho parcelas circulares de $15 \mathrm{~m}$ de radio, separadas entre sí por $60 \mathrm{~m}$ [16], para un total de 24 parcelas de muestreo (Figura 1). Dentro de cada una de estas se realizó la recolecta de las mariposas diurnas usando dos métodos: red entomológica y trampas Van Someren Rydon cebadas con una mezcla atrayente de banano, ron y jugo de caña, con el fin de atraer mariposas frugívoras, que incluyen especies típicas de zonas tropicales y subtropicales, principalmente mariposas de la familia Nymphalidae [17].

El muestreo con trampas se realizó durante cinco horas efectivas por parcela de muestreo, ubicadas a dos metros del suelo. Una vez instaladas, estas fueron visitadas dos veces al día para la recolección de los ejemplares capturados. La recolecta con red entomológica fue realizada entre las 8:00 -16:00 horas (debido a las condiciones logísticas del muestreo que no garantizaban la seguridad del personal de trabajo en campo) por tres personas en cada parcela, con un esfuerzo total de 5 horas de muestreo por parcela. Cabe destacar que todas las mariposas fueron recolectadas, pero solo se incluyeron en el análisis las mariposas pertenecientes a las familias Papilionidae, Pieridae, Lycaenidae, Riodinidae y Nymphalidae, dadas las dificultades en la identificación acertada de las especies de la familia Hesperiidae [18], lo cual puede llevar a subestimar su riqueza.

Una vez recolectado el material, este fue almacenado en sobres de papel milano [19], sobre los cuales fue consignada la siguiente información: número de parcela, datos de localidad (departamento, municipio), fecha, nombre del recolector, altitud, y coordenadas geográficas. El muestreo fue realizado en cinco salidas de campo con una duración de 8 días por salida entre los meses de Octubre-Noviembre del año 2014 y Abril-Mayo del año 2015 donde se presentaron los picos más altos de lluvias, que coincide con los picos poblacionales según Freitas, et al. [17] . 


\section{Identificación taxonómica}

Los ejemplares capturados fueron rehidratados en cámaras húmedas por un periodo mínimo de 24 horas para su ablandamiento y posterior montaje en el laboratorio de Conservación Biológica de la Universidad de Sucre. La extensión de las alas se realizó siguiendo el protocolo sugerido por Borror, et al. [19].

Una vez extendido el material se procedió a la identificación taxonómica, la cual consistió principalmente de dos procesos: (a) identificación mediante la comparación con fotografías de ejemplares tipo depositados en el sitio web "Butterflies of América" [20], guías de campo [21, 22] y check list [23]. (b) En el Museo entomológico de la Universidad Nacional de Colombia, sede Medellín, fueron verificadas las entidades taxonómicas. Finalmente, para aquellos individuos donde no fue posible una aproximación a una entidad taxonómica a través de morfología, fue necesario realizar disecciones de los órganos genitales usando una solución líquida de $\mathrm{KOH}$ al $10 \%$ en baño de maría durante 15 min siguiendo la metodología de Andrade $-\mathrm{C}$, et al. [24], para posterior observación en un estereoscopio Leika K100 (a 35X).

\section{ANÁLISIS DE DATOS}

Se realizaron curvas de rango-abundancia con transformación $\log (10)$ que permiten comparar la composición, abundancia y uniformidad de las especies en cada parcela [25]. Para calcular el número esperado de especies se realizó una curva de rarefacción y extrapolación-interpolación basado en la cobertura de la muestra utilizando el método descrito por Colwell, et al. [26]. Los análisis se realizaron con 100 aleatorizaciones y extrapolando al doble del número de individuos de la comunidad con la menor cobertura de muestra. Dichos análisis fueron implementados en la plataforma iNEXT disponible en https:// chao.shinyapps.io/iNEXTOnline.

\section{RESULTADOS}

En total se recolectaron 301 individuos de cuatro familias, que incluyeron 13 subfamilias,
22 tribus, 44 géneros y 55 especies (Tabla 1). La gráfica de rango-abundancia muestra que las mariposas tienen una estructuración representada por especies igualmente comunes y pocas especies dominantes. Dentro de los taxones con dominancia fueron encontradas Hamadryas februa (Hübner, [1823]) con un total de 56 individuos, seguida de Morpho helenor Cramer, 776 con 32 individuos. La completitud total del área de estudio fue de $89,04 \%$, esto significa que el muestreo fue representativo (Figura 3).

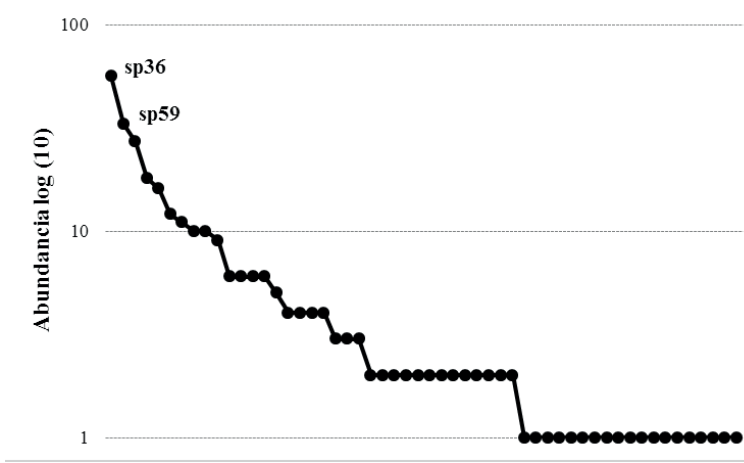

Figura 2. Gráfico de rango-abundancia de la comunidad de mariposas diurnas en la Reserva Forestal Protectora Serranía de Coraza. Sp36: Hamadryas februa; sp59: Morpho helenor.

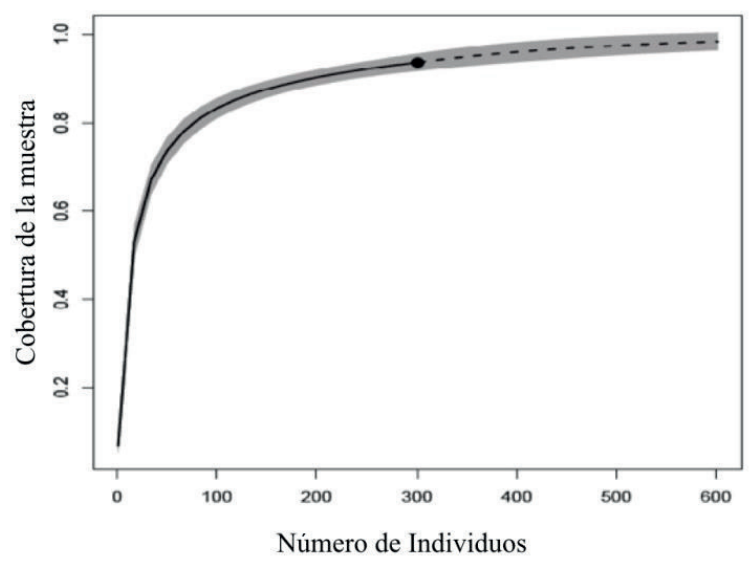

Figura 3. Curva de rarefacción y extrapolación basado en la riqueza de mariposas diurnas en la Reserva Forestal Protectora Serranía de Coraza. La línea sólida representa la cobertura de muestreo (interpolación) y la línea discontinua la extrapolación.

La familia más representativa en términos de abundancia fue Nymphalidae con 235 individuos, seguida de Pieridae, Papilionidae y Riodinidae (Figura 4a). En términos de riqueza los datos muestran el siguiente orden, Nymphalidae, Pieridae Papilionidae y Riodinidae (Figura 4b) como los grupos taxonómicos con más especies. 
Las subfamilias con mayor abundancia fueron Biblidinae Charaxinae, y Satyrinae (Figura 5a).
Del mismo modo Biblidinae presentó la mayor riqueza, seguida de Nymphalinae (Figura 5b).
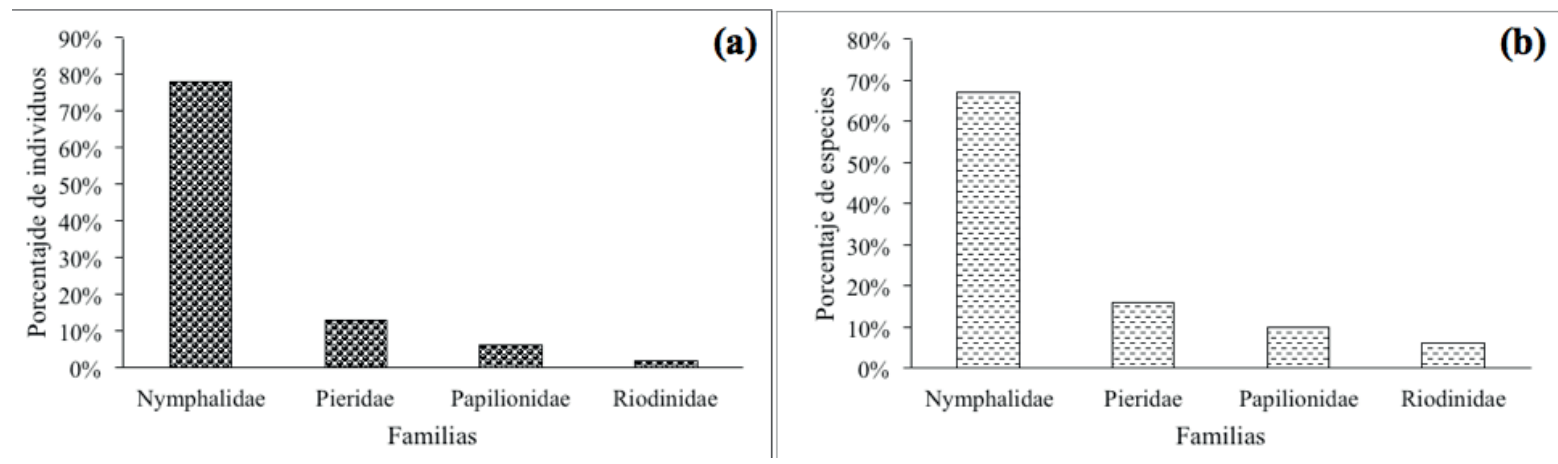

Figura 4. Abundancia y riqueza de familias en Montes de María. a) Representación de abundancia; b) Riqueza por familia de la comunidad de mariposas diurnas en la Reserva Forestal Protectora Serranía de Coraza.

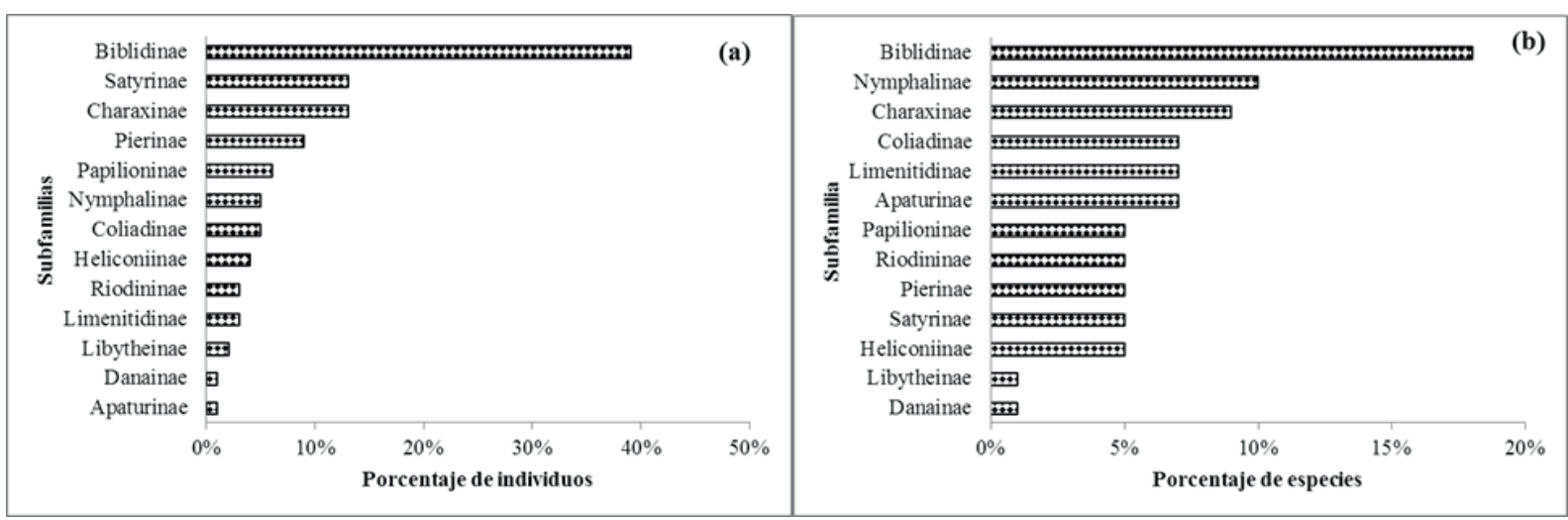

Figura 5. Abundancia y riqueza de subfamilias de mariposas en Montes de María. a) Representación de abundancia; b) Riqueza por subfamilia de la comunidad de mariposas diurnas en la Reserva Forestal Protectora Serranía de Coraza.

En las trampas Van Someren Rydon se capturaron 27 especies y 187 individuos, que represen$\tan$ el $49 \%$ y el $62 \%$ de la riqueza y la abundancia respectivamente. Las especies restantes fueron recolectadas utilizando red entomológica (Figura $6)$.

\section{DISCUSIÓN}

La completitud de los muestreos fue del $89,04 \%$, lo que significa que el esfuerzo de muestreo empleado fue suficiente para registrar la mayoría de las especies en el área estudiada. Caso contrario a lo reportado por Boom-Urueta, et al. [27] en la Reserva Ecológica Luriza (fragmento de bs-t, departamento del Atlántico), donde se observó que la curva de los estimadores no alcanzó la asíntota. Lo anterior demuestra la importancia del esfuerzo y técnica de muestreo (parcelas circulares) empleada en el presente estudio, lo que permitió abarcar más áreas con el mismo esfuerzo de muestreo en cada parcela, evitando sesgos en el método de captura.

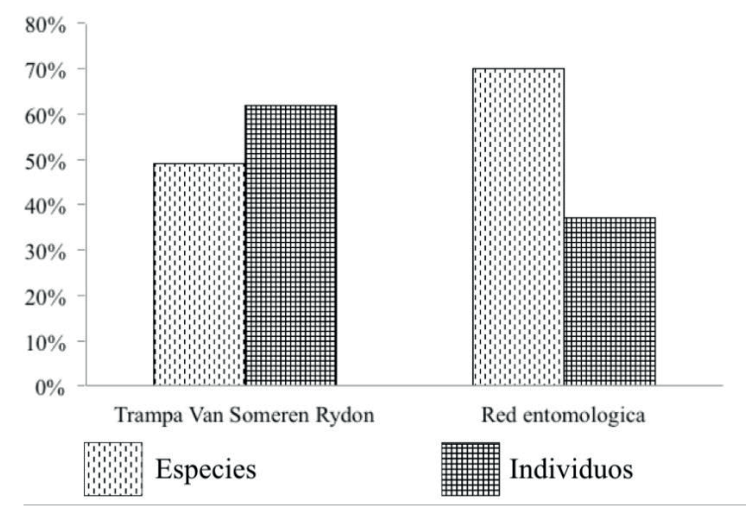

Figura 6. Porcentaje de especies y de individuos recolectados utilizando las Trampas Van Someren Rydon y Red entomológica.

Las 55 especies de mariposas registradas en este estudio son una muestra de su riqueza en los bosques secos tropicales de la reserva. A nivel de 
familias fueron encontradas cuatro (Nymphalidae, Papilionidae, Pieridae y Riodinidae) de las seis reportadas en Colombia por Montero, et al. [28], Orozco, et al. [29], Domínguez, et al. [8], Vargas-Zapata, et al. [30] y Boom-Urueta, et al. [27] en fragmentos de bs-t. Los datos obtenidos en términos de riqueza del presente estudio sumado a los resultados de otras investigaciónes [28, 31, 32], permiten sugerir que Nymphalidae, Pieridae, Papilionidae y Riodinidae como grupos dominantes en fragmentos de bs-t.

Tabla 1. Riqueza y abundancia de mariposas diurnas del bs-t de la Reserva Forestal Protectora Serranía de Coraza.

\begin{tabular}{|c|c|c|c|c|c|c|}
\hline Familia & Subfamilia & Tribu & Especie & $\begin{array}{c}\text { Red } \\
\text { entomológica }\end{array}$ & Trampas & Total \\
\hline \multirow[t]{29}{*}{ Nymphalidae } & Apaturinae & Biblidini & Doxocopa pavon theodora (Lucas,1857) & 0 & 1 & 1 \\
\hline & & Ageroniini & $\begin{array}{l}\text { Ectima erycinoides (C.Felder \& R. } \\
\text { Felder1867) }\end{array}$ & 0 & 1 & 1 \\
\hline & & & $\begin{array}{l}\text { Hamadryas februa (Hübner, [1823]) } \\
\text { (Godart,1824) }\end{array}$ & 0 & 56 & 56 \\
\hline & & & Hamadryas feronia (Linnaeus, 1758) & 0 & 12 & 12 \\
\hline & Biblidinae & Callicorini & Callicore pitheas (Latreille,1813) & 1 & 2 & 3 \\
\hline & & Catonephelini & Eunica tatila (Herrich-Schäffer, 1855) & 0 & 1 & 1 \\
\hline & & & $\begin{array}{l}\text { Myscelia leucocyana (C.Felder \& R. } \\
\text { Felder1861) }\end{array}$ & 1 & 26 & 27 \\
\hline & & Epiphelini & Nica flavilla (Godart,1824) & 1 & 17 & 18 \\
\hline & & & Pyrrhogyra neaerea (Menetries,1855) & 0 & 1 & 1 \\
\hline & & & Temenis laothoe (Cramer,1777) & 1 & 0 & 1 \\
\hline & & Anaeini & Consul fabius (A. Butler,1874) & 1 & 1 & 2 \\
\hline & & & Fountainea halice (Godart,1824) & 0 & 1 & 1 \\
\hline & & & Hypna clytemnestra (Cramer, 1777) & 0 & 4 & 4 \\
\hline & & & Memphis arginussa (Geyer,1832) & 0 & 2 & 2 \\
\hline & Charaxinae & & Siderone galanthis (Cramer,1775) & 1 & 5 & 6 \\
\hline & & & Zaretis ellops (Menetries,1855) & 0 & 16 & 16 \\
\hline & & Preponini & $\begin{array}{ll}\text { Archaeoprepona } & \text { demophon } \\
\text { (Linnaeus,1758) } & \end{array}$ & 0 & 6 & 6 \\
\hline & & & demophoon & 0 & 1 & 1 \\
\hline & & & Prepona laertes (Hubner,1811) & 0 & 2 & 2 \\
\hline & Danainae & Danaini & Lycorea halia (Hübner,1816) & 1 & 0 & 1 \\
\hline & Heliconiinae & Heliconiini & Dryas iulia (Fabricius,1775) & 1 & 0 & 1 \\
\hline & & & Heliconius erato (Linnaeus,1758) & 9 & 0 & 9 \\
\hline & & & Heliconius ethilla (Godart,1819) & 2 & 0 & 2 \\
\hline & Libytheinae & Stalachtini & Libytheana carinenta (Cramer,1777) & 0 & 2 & 2 \\
\hline & Limenitidinae & Limenitidini & Adelphafessonia ernestoi (Willmott,2003) & 2 & 0 & 2 \\
\hline & & & Adelpha iphicleola (H. Bates,1864) & 2 & 3 & 5 \\
\hline & & Melitaeini & Chlosyne lacinia (Geyer,1837) & 1 & 0 & 1 \\
\hline & & & $\begin{array}{l}\text { Chlosyne poecile (C.Felder \& R. } \\
\text { Felder1867) }\end{array}$ & 3 & 0 & 3 \\
\hline & Nymphalinae & & $\begin{array}{l}\text { Janatella leucodesma (C.Felder \& R. } \\
\text { Felder1861) }\end{array}$ & 6 & 0 & 6 \\
\hline
\end{tabular}


Yarlenis L. Mercado-Gómez et. al.

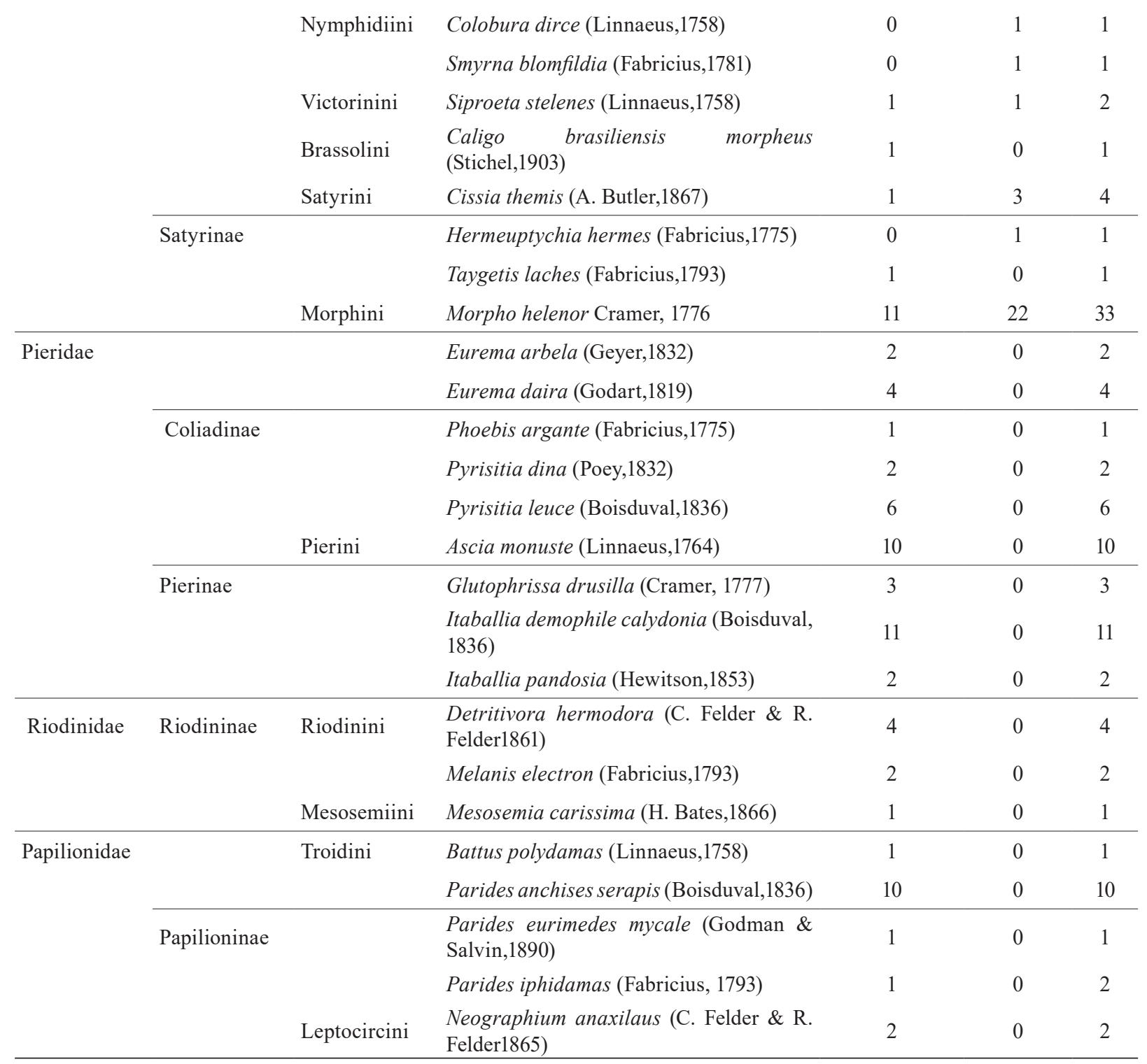

La familia Nymphalidae fue la más representativa en cuanto al número de individuos y especies, lo cual es coincidente con localidades de bs-t en el occidente antioqueño (municipio de San Jerónimo) donde Orozco, et al. [29], identificarón alrededor de 78 especies. En el departamento del Atlántico (localidad Corrales de San Luis) Prince-Chacón, et al. [33] registraron 38 especies, mientras en el departamento de Sucre, Domínguez, et al. [8] reportaron los valores más altos con 102 especies. La dominancia de este taxón puede estar relacionada a su amplio rango de distribución, diversidad de hábitos alimenticios y su capacidad de ocupar una gran variedad de hábitat que posiblemente mitigan competencia al interior del clado [33, 34].
La subfamilia Biblidinae presentó el mayor número de individuos, lo que puede estar relacionado con diferentes aspectos que mantienen poblaciones abundantes, facilitando su recolecta. En primer lugar, las plantas nutricias de sus larvas pertenecientes a las familias Malvaceae, Euphorbiaceae, Moraceae y Sapindaceae [35], que son típicas de estos bosques secos [36]. Estas ofrecen alimento y refugio para que las mariposas puedan completar su ciclo [33]. En segunda posición a la gran variedad de estrategias antidepredadoras y amplio periodo de vuelo [31], lo que les garantiza una mayor proliferación a lo largo del año.

La gran abundancia de la especie Hamadryas februa puede estar relacionada a sus hábitos alimenticios, debido a la preferencia que tienen por los frutos fermentados [37]. En este sentido, du- 
rante los periodos de recolecta las trampas fueron posicionadas dentro del bosque cerca de árboles en periodos de fructificación. Es importante aclarar que los frutos que no son dispersados durante este período, maduran rápidamente y caen al suelo en grandes cantidades, estos se fermentan atrayendo muchas especies de mariposa de este linaje (observaciones en campo). Las especies del género Hamadryas han sido reportadas por Montero, et al. [28] y Orozco, et al. [29] como individuos de amplia distribución y con una alta abundancia. En otras palabras, es posible que la alta abundancia de este género en la reserva, sea el producto tanto de la oferta alimenticia como de su rango de distribución.

Morpho helenor fue encontrada al interior del bosque y según Tavera [38] depende de bordes de quebradas e interior de bosque poco alterados debido a que presenta complejos requerimientos en el hábitat [39]. Bajo este contexto, es posible sugerir que los bosques secos de la reserva presentan condiciones ambientales propicias para el crecimiento y establecimiento de las poblaciones de este taxón. Es decir, dada las exigencias de esta especie se puede considerar que su presencia en los bosques secos de la reserva también indica el buen estado de conservación de los mismos.

Por otra parte, Domínguez, et al. [8] proponen una escala de abundancia según la metodología de Fagua [40], donde las especies con menos de tres individuos son consideradas raras y su ocurrencia está relacionada con periodos de vuelo discontinuos o emergencias alternas de imagos. Ellos encuentran [8] un total de 130 especies raras; mientras que, en el presente estudio de las 55 especies registradas para el bosque, 19 mostraron un número reducido de individuos. Por ejemplo, uno de los taxones considerados como raros es Callicore pitheas, lo cual también es reportado por Vargas-Zapata, et al. [30] en fragmentos de bs-t en el departamento del Atlántico.

Master [41] propone que la ocurrencia de las especies raras dentro del bosque puede indicar hábitat particulares o áreas de interés para la conservación, lo que sugiere que la reserva puede presentar fragmentos de bosques conservados. Del mismo modo, es posible que estas especies sean raras dado hacen parte de otras comunida- des de mariposas asociadas a otras coberturas vegetales como los potreros, bordes de bosque y cultivos. Por lo tanto, verlas al interior del bosque también puede ser un reflejo de la cercanía de sitios antropizados muy cercanos al bosque.

Las dos técnicas de muestreo empleadas para la recolecta de las mariposas fueron eficientes; sin embargo, la red entomológica arrojó mejores resultados, debido posiblemente a la facilidad de capturar mariposas en diferentes estratos vegetales. Según Domínguez, et al. [8] esta técnica permite capturar un gran número de especies con hábitos variados (zonas abiertas y cerradas) lo que permite maximizar la recolecta en el área de estudio.

\section{CONCLUSIONES}

Los resultados sugieren que los bosques secos de la Reserva Forestal Coraza, a pesar de estar actualmente sometidos a la minería indiscriminada, a cultivos y otros procesos antrópicos (ganadería y turismo) [12] poseen una gran riqueza de especies de mariposas. Los Montes de María abarcan un total de 6,653 ha, de las cuales aproximadamente 30 ha fueron muestreadas en el presente estudio, por lo cual las especies aquí reportadas podrían ser solo una muestra de la riqueza total de lepidópteros que puede ser hallada en la zona.

La presencia de especies raras en el bosque puede ser producto de la conservación de estos ecosistemas como bosque primario dentro de la reserva, o por la expansión de la actividad humana. Según lo anterior, es importante continuar realizando estudios en estos bosques, ampliando no solo el área y el espacio temporal de muestreo, también el alcance del mismo. Por ejemplo, usar la diversidad de mariposas del bosque seco para evaluar el efecto de las transformaciones antrópicas en la región. Es decir analizar su diversidad (alfa y beta) en ecosistemas poco alterados a muy transformados, como las áreas urbanas, cultivos y zonas de pastoreo. Este tipo de estudios permitiría identificar linajes típicos de los bosques secos de la región y la posible pérdida de los mismos ante escenarios de transformación antrópica. 


\section{AGRADECIMIENTOS}

A los habitantes del municipio de Colosó, en especial a Daniel Peralta por su ayuda incondicional en el desarrollo del trabajo de campo dentro de la Reserva, a mis colegas Pedro Álvarez y Ana Paola Mercado por su apoyo y acompañamiento durante los meses de muestreo. Al Laboratorio de Conservación Biológica de la Universidad de Sucre e Insectario de la Universidad Nacional, sede Medellín por facilitar las instalaciones para la identificación y almacenamiento de los ejemplares.

\section{REFERENCIAS}

[1] K. Banda, A. Delgado-Salinas, K. G. Dexter, R. Linares-Palomino, A. Oliveira-Filho, D. Prado, et al., "Plant diversity patterns in neotropical dry forests and their conservation implications," Science, vol. 353, pp. 1383-1387, 2016.

[2] R. Linares-Palomino, A. T. Oliveira-Filho, and R. T. Pennington, "Neotropical Seasonally Dry Forests: Diversity, Endemism, and Biogeography of Woody Plants. Seasonally Dry Tropical Forests," R. Dirzo, H. S. Young, H. A. Mooney, and G. Ceballos, Eds., ed: Island Press/Center for Resource Economics, 2011, pp. 3-21.

[3] H. García, G. Corzo, P. Isaac, and A. Etter, "Distribución y estado actual de los remanentes del bioma de bosque seco tropical en Colombia: Insumos para su conservación," in El bosque seco tropical en Colombia. vol. 90, C. Pizano and H. García, Eds., ed Bogotá, D.C.: Instituto de Investigación de Recursos Biológicos, Alexander von Humboldt, 2014, pp. 228-251.

[4] A. Etter, C. McAlpine, and H. Possingham, "Historical Patterns and Drivers of Landscape change in Colombia since 1500: A regionalized spatial approach," Annals of the Association of American Geographers, vol. 98, pp. 2-23, 2008.

[5] C. Pizano, R. González, M. González, R. Castro-Lima, N. Rodríguez, A. Idarraga, et al., "Plantas de los bosques secos de Colombia," in El bosque seco tropical en
Colombia, C. Pizano and H. García, Eds., ed Bogotá, D.C.: Instituto de Investigaciónes Alexander von Humboldt, 2014, pp. 37-48.

[6] M. Sampedro, H. Gómez, and G. Ballut, "Estado de la vegetación en localidades abandonadas por "desplazamiento, en los montes de María, Sucre, Colombia," RECIA, vol. 6, pp. 184-193, 2014.

[7] D. Olascuaga and J. Mercado-Gómez, "Análisis de la vegetación sucesional en un fragmento de bosque seco tropical en Toluviejo-Sucre (Colombia)," Colombia Forestal, vol. 19, pp. 23-40, 2016.

[8] K. Domínguez, J. González, and Y. Támara, "Composición y Abundancia de Mariposas Diurnas (Lepidoptera: Rhopalocera) en la Vegetación de Ribera del Arroyo el Sereno, Municipio de Colosó, Departamento de Sucre" Biología Universidad de Sucre, Sincelejo, 2011.

[9] K. Brown, "Conservation of Neotropical Enviroments: Insects as Indicators," in The Conservation of Insects and their habitat, $\mathrm{N}$. M. Collins and J. A. Thomas, Eds., ed New York: Academic Press, 1991, pp. 349 - 404.

[10] C. Kremen, R. Colwell, T. Erwin, D. Murphy, R. Noss, and M. Sanjayan, "Terrestrial arthropod assemblages: their use in conservation planning. ," Conservation Biology, vol. 7, pp. 796-808, 1993.

[11] J. Scott, The Butterflies of North America: A Natural History and Field Guide: Stanford, Un. Press, 1986.

[12] M. Aguilera, "Montes de María: Una subregión de economía campesina y empresarial," Documentos de trabajo sobre economía regional. Banco de la República, vol. 195, 2013.

[13] S. Galván-Guevara, M. Sierra, F. Gómez, J. De la Ossa, and A. Fajardo-Patiño, "Biodiversidad en el área de influencia de la estación primates de Colosó, Sucre, Colombia," RECIA, vol. 1, pp. 95-118, 2009.

[14] M. Aguilera, "La Economía del departamento de Sucre: Ganadería y Sector público," in Documentos de trabajo sobre 
economía regional, ed Cartagena de Indias: Banco de la República: Centro de Estudios Económicos Regionales (CEER), 2005.

[15] L. R. Holdridge, "Determination of World Plant Formations from Simple Climatic Data," Science, vol. 105, pp. 367-368, 1947.

[16] M. Elías, Z. Gompert, Jiggins, C., and W. Willmott, "Mutualistic Interactions Drive Ecological Niche Convergence in a Diverse Butterfly Community," PLoS Biol, vol. 6, pp. 300-308, 2008.

[17] L. Freitas, C. Iserhard, J. Santos, O. Junia, R. Danilo, A. Douglas, et al., "Studies with butterfly bait traps: an overview," Revista Colombiana de Entomología, vol. 40, pp. 203-212, 2014.

[18] M. González and M. Andrade - C., "Diversidad y biogeografía preliminar de las mariposas saltarinas (Lepidoptera: Hesperiidae) de Colombia," Rev. Acad. Colomb. Cienc vol. 32, 2008.

[19] D. Borror, C. Triplehorn, and N. Johnson, An Introduction to the Study of Insects. Philadelphia: Hartcourt Brace Jovanovich College, 1982.

[20] A. Warren, K. Davis, E. Stangeland, J. Pelham, K. Willmott, and N. Grishin. (2014). Butterflies of America." Retrieved Illustrated Lists of American Butterflies.

[21] J. Lecrom, L. Constantino, and J. Salazar, Mariposas de Colombia. Colombia: CARLEC Ltda, 2002.

[22] J. Lecrom, J. Llorente, L. Constantino, and J. Salazar, Mariposas de Colombia Tomo 2: Pieridae. Bogotá - Colombia: CARLEC Ltda, 2004.

[23] G. Lamas, Atlas of Neotropical Lepidoptera: Association for Tropical Lepidoptera, 2004.

[24] M. Andrade-C, E. Henao-Bañol, and P. Triviño, "Técnicas y Procedimientos para la Recolección, Preservación, y Montaje de Mariposas en Estudios de Biodiversidad y Conservación (Lepidoptera: Hesperioidea-Papilionoidea)," Revista Académica
Colombiana de Ciencias Naturales, vol. 37, pp. 311-225, 2013.

[25] A. Magurran, Ecological Diversity an its Measurement. New Jersey: Princeton Un.Press., 1988.

[26] R. Colwell, A. Chao, N. Gotelli, L. Shang-Yi, M. Chang Xuan, C. Robin L, et al., "Models and estimators linking individual-based and sample-based rarefaction, extrapolation and comparison of assemblages," Journal of Plant Ecology, vol. 5, pp. 3-21, 2012.

[27] C. Boom-Urueta, L. Seña-Ramos, M. Vargas-Zapata, and N. Martínez-Hernández, "Mariposas Hesperioidea y Papilionoidea (Insecta: Lepidoptera) en un fragmento de Bosque Seco Tropical, Atlántico, Colombia," bol.cient.mus.hist.nat, vol. 17, pp. 149-167, 2013.

[28] F. Montero, M. Moreno, and C. Gutiérrez, "Mariposas (Lepidoptera: Hesperioidea y Papilionoidea) asociadas a fragmentos de Bosque Seco Tropical en el departamento del Atlántico, Colombia," bol.cient.mus.hist. nat. , vol. 13, pp. 157 - 173, 2009.

[29] S. Orozco, S. Muriel, and J. Palacio, "Diversidad de Lepidópteros diurnos en una área de Bosque Seco Tropical del Occidente antioqueño.," Actual Biol, vol. 90, pp. 31 41, 2009.

[30] M. A. Vargas- Zapata, C. Boom-Urueta, L. Seña-Ramos, A. L. Echeverry-Iglesias, and N. J. Martínez- Hernández, "Composición Vegetal, Preferencias Alimenticias y Abundancia de Biblidinae (Lepidoptera: Nymphalidae) en un Fragmento de Bosque Seco Tropical en el Departamento del Atlántico, Colombia," Acta Biológica Colombiana, vol. 20, pp. 79-92, 2015.

[31] M. A. V. Zapata, N. J. M. Hernández, L. C. G. Moreno, S. P. Chacón, V. H. Colon, and L. F. T. Periñan, "Riqueza y Abundancia de Hesperioidea y Papilionoidea (Lepidoptera) en la Reserva Natural las Delicias, Santa Marta Magdalena, Colombia," Acta Biol. Colomb, vol. 16, 2011.

[32] L. Casas-Pinilla, J. Mahecha-J, C. Dumar-R, and I. Rios-Malaver, "Diversidad de mari- 
posas en un paisaje de Bosque Seco Tropical, en la Mesa de los Santos, Santander, Colombia. (Lepidoptera: Papilionoidea)," SHILAP Revista de Lepidopterología, vol. 45, pp. 83-108, 2017.

[33] S. Prince- Chacón, M. A. Vargas- Zapata, J. A. Salazar-E, and N. J. Martínez- Hernández, "Mariposas Papilionoidea y Hesperioidea (Insecta: lepidoptera) en dos fragmentos de Bosque Seco Tropical en Corrales de San Luis, Atlántico, Colombia," Boletín de la Sociedad Entomológica Aragonesa (S.E.A.), vol. 48, pp. 243-252, 2013.

[34] I. Chacón and J. Montero, Mariposas de Costa Rica: Costa Rica, 2007.

[35] J. M. Díaz, Bosque Seco Tropical en Colombia. Cali: Banco de Occidente, 2006.

[36] V. Herazo, H. Mendoza, and J. Mercado-Gómez, "Estructura y composición florística del bosque seco tropical en los Montes de María (Sucre - Colombia)," Ciencia en Desarrollo, vol. 8, 2017.
[37] C. García, L. Constantino, M. Heredia, and G. Kattan, Mariposas comunes de la cordillera central de colombia: WCS, 2002.

[38] S. Tavera, "Efecto de borde en la estructura y composicion de mariposas (Lepidoptera:Papilionoidea) de Bosque Andino en el sector occidental de la sabana de Bogotá," Universidad Javeriana, Bogotá, 2000.

[39] C. Millán, P. Chacón, and A. Giraldo, "Estudio de la comunidad de Lepidopteros diurnos en zonas naturales y sistemas productivos del municipio de Caloto (Cauca, Colombia)." Bol.cient.mus.hist.nat., vol. 13, pp. 185-195, 2009.

[40] G. Fagua, "Comunidad de mariposas y artropodofauna asociada con el suelo de tres tipos de vegetación de la Serranía de Taraira (Vaupés, Colombia). Una prueba del uso de mariposas como bioindicadores," Revista Colombiana de Entomología, vol. 22, pp. 143-151, 1996.

[41] L. L. Master, "Assesing treats and setting priorices for conservation," Conservation Biology, vol. 5, pp. 559-563, 1991. 
